\title{
Percepción estudiantil sobre una metodología asistida por computadora en las áreas cognitivas del álgebra lineal y la matemática discreta
}

\author{
Enrique Vílchez Q. \\ enrique.vilchez.quesada@una.cr \\ Universidad Nacional de Costa Rica
}

Elizabeth González S. elizabgonza@gmail.com Universidad Nacional de Costa Rica

Recibido: 20 Febrero 2013

Aceptado: 4 Junio 2013

\begin{abstract}
Resumen. Se presentan los resultados de una investigación de carácter descriptivo realizada sobre una muestra de 68 estudiantes inscritos en cursos vinculados con álgebra lineal y matemáticas discretas, específicamente en dos instituciones de enseñanza superior pública en Costa Rica: la Universidad Nacional y la Universidad de Costa Rica. El estudio pretendió analizar a través de la participación de tres grupos pilotos (dos de álgebra y uno de matemática discreta) el impacto en términos de enseñanza y aprendizaje de la implementación de una metodología asistida por computadora, recurriendo al uso del software Mathematica como principal agente mediador de experiencias de aprendizaje. En los grupos pilotos se aplicó la experiencia docente durante el II semestre 2012 y al finalizar este período, se solicitó a la población estudiantil llenar un cuestionario de percepción. En la presente propuesta se analizan los resultados más significativos de esta experiencia, sistematizando algunas recomendaciones esenciales para replicar otras metodologías semejantes en diversos contextos.
\end{abstract}

Palabras clave: matemática, software, metodología, discretas, álgebra

Abstract. we present the results of a descriptive study which sought to analyze through the participation of three pilot groups (two of algebra and one of discrete mathematics), the teaching and learning impacts resulting from the implementation of a computer-assisted methodology, using Mathematica software application as the main mediator of learning experiences. The study was conducted on a sample of 68 students enrolled in courses related to linear algebra and discrete mathematics, specifically on two public higher education institutions in Costa Rica: National University and the University of Costa Rica. The teaching experience was carried out during the second semester 2012. Afterwards, the participating students were asked to fill'out a perception questionnaire. This proposal analyzes the most significant results of this experience, systemize a number of essential steps to replicate other similar methodologies in different contexts. 
KeyWords: mathematics, software, methodology, discrete, algebra

\subsection{Introducción}

La matemática asistida por computadora es un área de investigación que busca propiciar espacios de análisis sobre el uso de distintas tecnologías para el desarrollo de procesos de enseñanza y aprendizaje vinculados con esta disciplina científica. Astorga, A. y Sánchez, A (1999) señalan cómo este enfoque metodológico ha intentado buscar nuevas opciones didácticas que contribuyan con la educación matemática en sus distintas áreas de conocimiento:

“Una respuesta a la problemática de la matemática y su enseñanza lo ha aportado el paradigma de los procesos de aprendizaje asistidos por computadora, el cual en los últimos años ha recibido bastante análisis y sustento teórico de disciplinas como la psicología, inteligencia artificial, pedagogía, etc" (p. 198).

En Costa Rica desde el año 1980 se han venido implementado distintas experiencias de formación hacia la búsqueda de nuevos ambientes de aprendizaje, basados en el uso de recursos computacionales y calculadoras de última generación (De Faria, 2006). Más recientemente, han aumentado en este escenario acciones concretas impulsadas por los nuevos modelos pedagógicos en un sistema educativo, que apuesta en las tecnologías digitales una opción viable para implementar acciones que disminuyan el fracaso escolar en matemáticas, demostrado por la población estudiantil en las pruebas estandarizadas.

Esta visión entusiasta proviene de un sistema económico donde los gastos crecientes en tecnologías de información y comunicación, está superando las expectativas de beneficio iniciales en Latinoamérica. En México por ejemplo, el proyecto "Enciclomedia" implicó un gasto nacional aproximadamente de 1100 millones de dólares (Piscitelli, 2010) que de acuerdo con los diagnósticos técnicos realizados en la región, no logró plasmar sus objetivos de implementación por dos razones principales: los docentes muestran aún una significativa resistencia al uso de las tecnologías y las aplicaciones educativas hoy por hoy, están muy lejos de igualar el atractivo hipnótico de los video juegos con todas sus variaciones mediáticas.

Lo anterior no representa un menosprecio a las ventajas que podrían ofrecer los ambientes de aprendizaje basados en el uso de la computadora y una enseñanza bien articulada. Lo fundamental es comprender que las tecnologías no son una respuesta mágica ante los desafíos del desarrollo cultural, económico o social, pues mal empleadas pueden producir efectos tan desastrosos como cualquier otro medio de movilidad.

Esta advertencia a nuestro juicio, debe ser el punto de partida de cualquier proceso de innovación y en el campo educativo se constituye en una invitación al establecimiento de mecanismos que logren sistematizar las mejores prácticas. La presente investigación tuvo esta orientación, con una visión neutral sobre los beneficios o limitaciones del conocido software comercial Mathematica para potencializar la enseñanza y el aprendizaje del álgebra lineal y las matemáticas discretas. Bajo esta perspectiva, el presente estudio tuvo como principal objetivo, obtener una percepción estudiantil sobre el uso de una metodología asistida por computadora en estas dos áreas de conocimiento. 


\subsection{Enseñanza asistida por computadora}

Enseñar de forma asistida por computadora va más allá del simple aunque costoso hecho, de tener las computadoras y el software necesario. Córica y Dinerstein lo advierten:

“(...) el solo hecho de descargar millones de computadoras en las aulas, no va a remediar los males que aquejan a la educación contemporánea, así como no la remediaban llevar una selección aleatoria de libros a las escuelas o prescribir medicamentos al azar" (2009, p. 70).

La intencionalidad didáctica circunscribe varias dimensiones simultáneamente: la metodológica, los recursos materiales y la evaluativa.

En cuanto a la metodología, De Faria señala: "no tiene ningún sentido utilizar una calculadora o una computadora para seguir haciendo las mismas cosas que hacíamos con las herramientas tradicionales: lápiz, papel, tiza y borrador" (2006, p. 711). Como bien lo apunta este autor, un cambio metodológico no significa trasladar las mismas prácticas de enseñanza a otros medios. Una computadora tiene la capacidad de resolver en segundos lo que posiblemente tardaríamos horas en realizar. Esta clara ventaja, demanda objetivos educativos asociados no tanto con la resolución de problemas algorítmicos, sino más bien, con la exploración, análisis, búsqueda e investigación. Los nobles principios educativos anteriormente citados, muchas veces no son fáciles de conciliar en una realidad contextual que encuentra más comodidad en la repetición y reproducción cognitiva. Gardner (2001) así describe: "Es probable que recurrir a esta actuación provoque estrés en los estudiantes, los enseñantes y los padres, que se han acostumbrado a las maneras tradicionales de hacer o no hacer las cosas" (p. 500).

La construcción personal o colectiva del conocimiento no suele ser una competencia del estudiantado que ingresa a la Universidad. Naturalmente, los alumnos fieles a su tradición de aprendizaje, entran en conflicto directo con aquellas estrategias de enseñanza que los desvinculan de la escolaridad y estandarización típicas en las instituciones educativas. Esto significa que los cambios metodológicos deben ser graduales y en consistencia con las características de sus actores, de lo contrario, la frustración y el agobio conducirán muy posiblemente, hacia el fracaso académico.

Es curioso en este punto, observar cómo los jóvenes frecuentan las tecnologías digitales en su vida diaria, sin embargo, hay una disrupción cuando ellas forman parte de sus actividades escolares. La explicación a este fenómeno se encuentra ligada a la alfabetización digital. Alfabetizar digitalmente no implica solo enseñar a utilizar las herramientas proveídas por las tecnologías, es un desafío más supremo, relacionado con su incorporación transparente en la productividad cognitiva. A este respecto Coicaud menciona:

"Desde una postura diferente, cuando los docentes atribuyen otro tipo de significaciones a la tecnología, convirtiéndola en una herramienta válida que posibilita operar con los conocimientos a partir de múltiples sentidos, se generan actitudes de involucramiento y compromiso con la tarea por parte de los alumnos, quienes aprenden a moverse dentro de una gran variedad de desempeños de comprensión que van adquiriendo una complejidad creciente" (2010, p. 59).

Educación con y de las tecnologías, por lo tanto, atañe al campo de la metacognición en procesos guiados donde el estudiante aprende a aprender. 
Los recursos materiales constituyen un elemento importante en la educación mediada, los medios en sí mismos son recursos y dentro de ellos a su vez, se utilizan materiales. Años atrás la generación de recursos educativos demandaba esfuerzos que transcendían las posibilidades reales dentro del quehacer de los grupos de docentes encargados de gestar los aprendizajes. Actualmente, la reutilización de objetos de aprendizaje, bibliotecas digitales, podcasts, multimedios, juegos, software y una gama diversificada de otras fuentes disponibles en la red, está dotando a las instituciones de enseñanza de importantes oportunidades de formación online. Por ejemplo, la compañía Wolfram Research encargada del desarrollo del software Mathematica, recientemente ha puesto en línea el sitio: http://www. wolframalpha. com que integra un programa denominado Wolfram | Alpha. Esta aplicación es capaz de responder en tiempo real preguntas de carácter matemático, brindando múltiples configuraciones de respuesta. Para un docente de matemáticas podría ser interesante estudiar las posibilidades didácticas de Wolfram $\mid$ Alpha, dada su naturaleza abierta y de bajo costo. Este es uno de los retos más significativos en la nueva dimensión tecnológica que enfrentan los educadores, estudiar las posibilidades de uso de recursos que inclusive no fueron creados con una intencionalidad pedagógica. Entender la funcionalidad de estos materiales puede convertirse en un hilo orientador crucial, para juzgar sus posibilidades de incorporación responsable y planificada en el aula. Según Córica y Dinerstein (2009) las funcionalidades de estos medios o recursos se clasifican en: proporcionar información, guiar los aprendizajes de los estudiantes, ejercitar habilidades, mantener el interés, evaluar los conocimientos, proporcionar simulaciones que ofrecen entornos para la observación, exploración y la experimentación y, proporcionar entornos para la expresión y creación.

La evaluación en la enseñanza asistida por computadora es otro tema notable. Una propuesta de innovación justa no solo debe enfocarse en los cambios metodológicos y los recursos necesarios, debe ser partícipe de un proceso de reflexión donde el nivel de avance de los estudiantes sea valorado más allá de las pruebas estandarizadas en papel. De Faria cita: "Estamos convencidos de que sin un cambio (...) en la forma de evaluar, no podemos esperar cambios en las actitudes, aprendizajes y promociones de los estudiantes" (2006, p. 712). En el marco de la presente investigación, se promovió un esquema de evaluación asistida por computadora en dos sentidos: por un lado los estudiantes podían utilizar el software Mathematica en la resolución de los problemas propuestos en las evaluaciones y por otro, el nivel de dificultad de algunas de las preguntas exigía la asistencia del ordenador.

\subsection{Aprendizaje estimulado a través del uso de software}

Desde hace varios años el software educativo es una de las tendencias que intentan promover cambios significativos en la calidad de la educación en general y específicamente en la enseñanza de las matemáticas. Una prueba de ello, radica en la aparición de distintas aplicaciones de software libre y licenciado que pretenden fortalecer diversos procesos de enseñanza y aprendizaje en campos específicos y generales, algunas de ellas son: Mathematica, Matlab, Fathom, TinkePlots, Skechpad, Geometer Expressions, WinPlot, entre otras.

El software con fines didácticos trasluce una incitación pedagógica en matemáticas, donde los cálculos mecánicos ausentes de un análisis real en la resolución de problemas, pasan a un segundo plano. Lo importante en esta dirección, es el diseño de las experiencias de aprendizaje en un marco de estrategias metodológicas, donde el software consolide las aristas necesarias que interconecten la creatividad 
del estudiante con sus conocimientos teóricos. Lo anterior hace emerger la aplicación para dar una significación más amplia a lo que se aprende. Gamboa reafirma esta concepción:

"La función del educador es ofrecer, a través del diseño de una situación, un encuentro entre el sujeto y el medio para que surja el conocimiento. En este sentido, el empleo de herramientas tecnológicas debe ir orientado a apoyar y contribuir para que el sujeto construya, adecuadamente, diferentes representaciones con el fin de modificar los antiguos sistemas de percepción y, con ello, el surgimiento de su conocimiento" (2007, pp. 19-20).

Nuevas formas de representación de ese conocimiento conforma una de las ventajas asociadas al uso apropiado en el aula, de los programas computacionales. Una animación o movilidad de objetos matemáticos realizada con software, puede brindar aclaraciones conceptuales que posiblemente apoyadas con otros medios, son muy difíciles de replicar. Alpízar comparte una idea similar:

“La presencia de la tecnología en el aula se convierte es una herramienta capaz de aportar a las clases de matemáticas sistemas de representación que puedan ser utilizados para la visualización y experimentación de conceptos importantes, lo que contribuye con estrategias para la resolución de problemas" (2007, p. 101).

Esta investigación parte de la premisa de considerar el uso de software como un apoyo didáctico fundamental en la encrucijada del aprendizaje, a través del uso de un sistema de cálculo simbólico, como lo constituye el software Mathematica. Nuestras consideraciones son apoyadas por De Faria al expresar:

“Los sistemas de cálculo simbólico (Computer algebra systems, CAS) posibilita la definición, manipulación, transformación, comparación y visualización de expresiones algebraicas en todos los registros de representaciones tradicionales y, además, facilita el tratamiento entre los distintos registros. Estas son características importantes en la modelación matemática, la simulación y en la resolución de problemas" (2007, p. 129).

Mathematica bajo esta perspectiva, es una opción viable de aprendizaje en muy diversas áreas de conocimiento, a través de una metodología asistida por computadora.

\subsection{El software Mathematica}

El software Mathematica fue creado por Stephen Wolfram, un genio contemporáneo graduado como físico de partículas del Instituto de Tecnología de California (Caltech). Desde el año 1988 ha venido liderando la compañía Wolfram Research encargada del desarrollo y comercialización del programa. En la actualidad Mathematica es utilizado en 15 departamentos principales del gobierno de los Estados Unidos y en 50 de las universidades más grandes del mundo. Esto refleja en alguna medida, la importante influencia de la aplicación para el desarrollo científico.

Mathematica es un software de cálculo simbólico que cuenta con diversas funcionalidades para trabajar en campos tales como: matemática, física, biología, ciencias sociales, ciencias de la computación, entre otras. En el ámbito educativo está ocupando un lugar cada vez más preponderante tanto a un nivel medio como superior. Las ofertas educativas a nivel mundial se han venido diversificando, incluyendo el uso de la aplicación en cursos de secundaria, universitarios y de postgrado. Wolfram Research (2013) 
afirma que es uno de los programas por excelencia, utilizado por múltiples profesionales técnicos y más recientemente, profesionales relacionados con el quehacer educativo.

\subsection{Implementación de una experiencia docente}

En esta sección se explican los aspectos metodológicos principales que caracterizaron el presente estudio.

\subsubsection{Antecedentes}

En el II semestre del año 2012 se implementó una experiencia docente a través de tres grupos pilotos: uno de Matemática III para Informática (álgebra lineal), otro de Estructuras Discretas para Informática (matemática discreta) ambos pertenecientes al plan de estudios de la carrera Ingeniería en Sistemas de Información de la Universidad Nacional de Costa Rica (UNA) y finalmente, un curso de Álgebra Lineal para Ingeniería propio de la Universidad de Costa Rica (UCR). Los grupos fueron conformados de forma aleatoria por la matrícula institucional de cada universidad. La Universidad Nacional y la Universidad de Costa Rica son instituciones públicas de enseñanza superior con un alto renombre en esta nación.

El objetivo de esta iniciativa se circunscribió determinar la percepción de los estudiantes ante el desafío del uso de software como apoyo para desarrollar procesos de enseñanza y aprendizaje vinculados con áreas cognitivas de carácter matemático. Particularmente, se recurrió al uso del software Mathematica como principal agente mediador en esta estrategia asistida por computadora. Se tomó la decisión de utilizar Mathematica como una herramienta de apoyo para desarrollar procesos de enseñanza y aprendizaje vinculados con matemática discreta y álgebra lineal, por la especialización de instrucciones y comandos que incorpora el software en estos campos de estudio y de la misma forma, por el hecho de contar con una licencia institucional comprada por la Universidad Nacional de Costa Rica y la Universidad de Costa Rica.

\subsubsection{Población participante en el estudio}

Se consideró una población de 68 estudiantes inscritos en cursos vinculados con álgebra lineal y matemáticas discretas, específicamente en dos instituciones de enseñanza superior pública en Costa Rica: la Universidad Nacional y la Universidad de Costa Rica. De ellos $18 \%$ fueron alumnos de género femenino y un $82 \%$ de género masculino en un rango de edades entre los 17 y 27 años.

\subsubsection{Procedimiento seguido}

La metodología de los tres cursos se modificó significativamente en comparación con la clase magistral tradicional utilizada durante años. El escenario de aula se sustituyó por un laboratorio de informática en un ámbito presencial y el uso de un aula virtual que en esta ocasión sirvió como un repositorio de materiales y seguimiento de noticias. El profesor complementaba en las sesiones presenciales, las explicaciones teóricas de la materia con ejemplos resueltos en la pizarra y posteriormente resueltos a 
través del uso del software Mathematica. Al principio de semestre los ejemplos propuestos fueron de naturaleza de cálculo y poco a poco se subió el nivel de dificultad de los problemas hasta llegar a situaciones de aprendizaje donde el alumno debía formular una conjetura ante un enunciado propuesto. Por ejemplo, para el curso de álgebra lineal en el tema de matrices una pregunta resulta en clase fue la siguiente:

Halle una conjetura que exprese la inversa de la matriz $n \times n$

$$
A=\left(\begin{array}{ccc}
1 & 1 & 1 \cdots 1 \\
0 & 1 & 1 \cdots 1 \\
0 & 0 & 1 \cdots 1 \\
\vdots & \vdots & \ldots \vdots \\
0 & 0 & 0 \cdots 1
\end{array}\right)
$$

El proceso de resolución implicaba el diseño de una serie de experimentos que le permitieran al estudiante visualizar algunos casos particulares y mediante sus observaciones, proponer la forma de la inversa de tamaño $n x n$. Estas situaciones de aprendizaje en su totalidad fueron tomadas del libro "Álgebra lineal apoyada con Mathematica" (Vílchez, 2012).

En el curso EIF-203 Estructuras Discretas para Informática, se recurrió a una serie de experiencias de aprendizaje formuladas en un texto titulado: "Estructuras discretas con Mathematica" (Vílchez, 2013). Un ejemplo de desarrollo en el tema de Relaciones durante la dinámica de la clase fue:

Ejemplo 38 Determine, con apoyo de software, el dominio y rango de la relación $R$ dada por: a $R b$ si y sólo si el máximo común divisor entre a y $b$ es igual a uno, con $a \in\{1,3,5,7\}$ y $b \in\{2,4,6,8\}$. ¿Cuáles valores del máximo común divisor satisfacen que la relación $R$ es distinto del conjunto vacío?

En él, nuevamente se propone la necesidad de usar software como un mecanismo de investigación.

Lo más trascendental de la experiencia fue el cambio de mentalidad que implicó tanto a nivel administrativo como académico. Uno de los autores de este artículo, mediante el uso de una metodología de investigación de carácter cualitativo, asumió el rol de observador participante tanto en los grupos involucrados como en el área administrativa de ambas universidades. El uso de una bitácora por sesión, le permitió identificar categorías donde se vislumbra aún en el ámbito universitario, un recelo inmerso en un currículum oculto vinculado con el uso de las tecnologías. Aunque parezca contradictorio con los modelos pedagógicos en ambas instituciones públicas (Universidad Nacional de Costa Rica y la Universidad de Costa Rica), existe una clara preocupación por parte de las autoridades universitarias involucradas en este estudio, en poner en riesgo la calidad, la rigurosidad y el nivel de exigencia de los cursos, al utilizar metodologías de enseñanza y aprendizaje que entran en concordancia con una matemática asistida por computadora.

Los grupos participantes de esta experiencia, semanalmente convergían en dos clases presenciales donde el docente se encargó de explicar el contenido teórico de cada una de las unidades temáticas. En álgebra lineal se cubrieron los siguientes temas: matrices y determinantes, vectores, rectas y planos, espacios vectoriales, proyecciones ortogonales, transformaciones lineales y, diagonalización de matrices y operadores. En matemática discreta se abordaron los contenidos de: inducción, conteo, relaciones 
de recurrencia, análisis de algoritmos, relaciones, teoría de grafos, teoría de árboles, máquinas y autómatas de estado finito, máquinas de Turing y, lenguajes y gramáticas.

\subsubsection{Recursos, materiales utilizados y metodología asistida por computadora}

En cada sesión el profesor abordó la teoría y la práctica utilizando como apoyo los textos: “Álgebra lineal apoyada con Mathematica" (Vílchez, 2012) y "Estructuras discretas con Mathematica" (Vílchez, 2013), ambos documentos se caracterizan por estar estructurados con una clara metodología asistida por computadora, integrando experiencias de aprendizaje de tipo laboratorio. Los ejemplos que caracterizan a estas obras recurren al uso de software como un medio de exploración y construcción de conjeturas. Además, a través de los sitios web: http://www.escinf.una.ac.cr/mathematica y http://www.escinf.una.ac.cr/discretas se pusieron a disposición de los estudiantes una serie de materiales complementarios, entre ellos: los archivos fuentes de cada una de las situaciones de aprendizaje inmersas en los libros de texto citados, cuadernos interactivos (para el caso del curso de Estructuras Discretas), animaciones (pruebas cortas interactivos y un glosario de comandos), paquetes de Mathematica (en el campo de los autómatas y lenguajes) y software gratuito (el cuál era opcional y complementario al uso de Mathematica). Con relación al licenciamiento de Mathematica, tanto la Universidad Nacional de Costa Rica como la Universidad de Costa Rica, pagan anualmente una licencia institucional que les ofrece el privilegio de poner a disposición de cualquier estudiante y docente el poder acceder a este software.

En el II semestre 2012, profesor y alumnos fueron desarrollando la teoría de cada curso mediada con prácticas de laboratorio. A este respecto, los laboratorios consistieron en situaciones de aprendizaje que implicaban el uso de métodos de exploración e investigación en la resolución de problemas con un interesante desafío, la construcción de conjeturas. Los problemas por lo tanto, no se reducían a un cálculo inmediato, los estudiantes a través del ambiente de programación de Mathematica y sus funcionalidades traducidas en el uso de comandos, intentaban analizar cómo podían obtener conclusiones generales. Desde nuestro punto de vista, esta práctica es fundamental en la educación matemática, si se piensa en esta ciencia como un área de conocimiento que se ha desarrollado a través de su historia, tomando como base razonamientos de carácter inductivo y no deductivo.

La matemática en los libros de texto suele ser expuesta de forma deductiva, sin embargo, su construcción, elaboración y evolución disciplinar tienen una fuerte tendencia experimental en casos particulares, así es como se origina una teoría desde nuestro punto de vista. En el plano educativo, colocar al alumno en situaciones de aprendizaje que lo hagan vivenciar estas formas de construcción conceptual, pone en práctica lo mencionado por Coicaud como involucramiento y compromiso en la realización de una tarea. Nuestra propuesta metodológica se sustentó en este principio.

El uso de software y su consecuente derivación en construcciones conceptuales y aplicaciones de contenido, no es en un inicio una meta fácil de consagrar. Muchas veces en el discurso político e inclusive en la literatura, se alude a la resistencia mostrada por los docentes en cuanto al uso de la tecnología y no negamos este hecho, pero reconocemos en esta dinámica otro importante actor que muestra cierta reticencia: "el alumno". Puede que el estudiante no se resista al uso de la tecnología con un fin en sí misma, sin embargo, su amenazada holgura se manifiesta al detonarse las implicaciones que tiene una metodología que demanda no solamente la retención y recuperación. En este sentido, también la población estudiantil muestra resistencia al uso de la tecnología, cuando ella impone la necesaria 
resolución de problemas con un nivel de dificultad mayor, en comparación con los problemas que se resolvían antes de la aparición del software. Este es un primer factor que vivenciamos con esta experiencia: un buen diseño pedagógico asistido por computadora debe tomar en cuenta la inmersión tecnológica no solamente en la tecnología per se, sino en las competencias que el alumno posee y se espera adquiera en el proceso educativo.

La evaluación de los cursos también tuvo algunos cambios relevantes específicamente en cuanto a la asignación de micro tareas programadas y al uso del software Mathematica en la aplicación de los exámenes parciales. En los grupos de la Universidad Nacional de Costa Rica, los estudiantes resolvieron en su totalidad los exámenes con apoyo de software. En la Universidad de Costa Rica en cambio, se tomó la decisión en mutuo acuerdo con la coordinación académica, de eliminar una pregunta de cada parcial para ser sustituida por otra, que debió ser resuelta en el laboratorio de informática.

La nueva metodología y el sistema de evaluación empleado hizo emerger una hipótesis no comprobada por el tamaño y objetivo de este estudio, al parecer los cambios suscitados con este enfoque asistido por computadora tuvieron un impacto directo en el rendimiento académico específicamente en los grupos de álgebra lineal. En uno de ellos (UNA), un 88.88\% de los estudiantes aprobaron con éxito la materia (16 de 18 alumnos) y en el otro (UCR) un 75\% de los alumnos que finalizaron el curso (3 de 4), de un total de 7 estudiantes que lo iniciaron), lo aprobaron. En el grupo de la Universidad Nacional de Costa Rica, se destaca el hecho de haber contado con una población estudiantil con altos índices de repitencia: tres alumnos lo llevaban por tercera vez, tres por cuarta ocasión y cinco por sexta vez. El cambio metodológico creemos tuvo una fuerte incidencia en su nivel de motivación y comprensión de los contenidos, permitiendo que un $100 \%$ de estos estudiantes avanzaran con su carrera.

En el grupo del curso Estructuras Discretas para Informática, los resultados no fueron tan favorables en cuanto a rendimiento académico, solamente un $52 \%$ de los alumnos aprobaron. Lo anterior, no brinda ninguna información adicional pues normalmente el curso posee estos índices de reprobación. Creemos, que la razón de este resultado obedeció al nivel de exigencia del curso asistido por computadora y el tipo de competencias preliminares de los estudiantes. Nuestro supuesto obedece al observar en el transcurso del semestre el avance de los alumnos que en muchos casos, se les dificultó enormemente encarar las responsabilidades asignadas fuera de las horas de clase presenciales.

Al finalizar el II ciclo lectivo 2012, se solicitó a los participantes en los tres grupos piloto, llenar un cuestionario puesto en línea (ver anexo), con la intención de recabar información general sobre su nivel de satisfacción y rescatar las principales percepciones de esta experiencia de implementación. Los participantes del estudio tuvieron la guía del docente para dar significado a cada una de las preguntas realizadas, dado que el mismo profesor se encargó de administrar el instrumento de investigación en un laboratorio de informática. En la siguiente sección se muestran algunos de los resultados obtenidos.

\subsection{Resultados}

Con respecto a los participantes de esta prueba piloto, en la siguiente tabla se resumen los principales resultados: 


\begin{tabular}{|c|c|c|c|c|c|}
\hline $\begin{array}{l}\text { Eficiencia del uso de } \\
\text { software }\end{array}$ & $\begin{array}{c}1 . \\
\text { Muy de } \\
\text { acuerdo }\end{array}$ & $\begin{array}{c}2 . \\
\text { De acuerdo }\end{array}$ & $\begin{array}{c}3 . \\
\text { Medianamente } \\
\text { de acuerdo }\end{array}$ & $\begin{array}{c}4 . \\
\text { En } \\
\text { desacuerdo }\end{array}$ & $\begin{array}{c}5 . \\
\text { Muy en } \\
\text { desacuerdo }\end{array}$ \\
\hline $\begin{array}{l}\text { 1. Los procesos de } \\
\text { enseñanza mejoraron } \\
\text { con el uso de soft- } \\
\text { ware. }\end{array}$ & $29.09 \%$ & $34.55 \%$ & $27.27 \%$ & $5.45 \%$ & $3.64 \%$ \\
\hline $\begin{array}{l}\text { 2. Los procesos de } \\
\text { aprendizaje no mejo- } \\
\text { raron con el uso de } \\
\text { software. }\end{array}$ & $3.64 \%$ & $14.55 \%$ & $23.64 \%$ & $49.09 \%$ & $9.08 \%$ \\
\hline $\begin{array}{l}\text { 3. El software facil- } \\
\text { itó la resolución de } \\
\text { problemas. }\end{array}$ & $50.91 \%$ & $23.64 \%$ & $12.73 \%$ & $10.91 \%$ & $1.81 \%$ \\
\hline $\begin{array}{l}\text { 4. Prefiero que se } \\
\text { use una metodología } \\
\text { más tradicional al } \\
\text { impartir el curso. }\end{array}$ & $9.09 \%$ & $12.73 \%$ & $34.55 \%$ & $36.36 \%$ & $7.27 \%$ \\
\hline $\begin{array}{l}\text { 5. No considero que } \\
\text { el software Mathe- } \\
\text { matica ayude en los } \\
\text { procesos de investi- } \\
\text { gación (exploración y } \\
\text { construcción de con- } \\
\text { jeturas). }\end{array}$ & $7.27 \%$ & $3.64 \%$ & $21.82 \%$ & $52.73 \%$ & $14.54 \%$ \\
\hline $\begin{array}{l}\text { 6. El software se } \\
\text { convirtió en un ob- } \\
\text { stáculo en mi apren- } \\
\text { dizaje. }\end{array}$ & $7.27 \%$ & $7.27 \%$ & $21.82 \%$ & $50.91 \%$ & $12.73 \%$ \\
\hline $\begin{array}{l}\text { 7. El curso no mejoró } \\
\text { en cuanto a calidad } \\
\text { académica al utilizar } \\
\text { una metodología } \\
\text { asistida por com- } \\
\text { putadora. }\end{array}$ & $7.27 \%$ & $12.73 \%$ & $34.55 \%$ & $34.55 \%$ & $10.90 \%$ \\
\hline $\begin{array}{l}\text { 8. Nunca habia } \\
\text { utilizado software } \\
\text { para complemen- } \\
\text { tar los procesos } \\
\text { de enseñanza y } \\
\text { aprendizaje. }\end{array}$ & $43.64 \%$ & $25.45 \%$ & $9.09 \%$ & $16.36 \%$ & $5.46 \%$ \\
\hline
\end{tabular}

Tabla 1.1

En la tabla se observa una tendencia a considerar que los procesos de enseñanza mejoraron con el uso de software. Un 63.64\% de la población estudiantil, estuvo "Muy de acuerdo" y "De acuerdo" con esta afirmación. Además, $58.17 \%$ de los alumnos participantes opinaron que los procesos de aprendizaje mejoraron con el uso de software. Si bien es cierto, la enseñanza y el aprendizaje son procesos separados, se vinculan constantemente en distintos espacios tanto de educación formal como informal. Estos porcentajes evidencian una aceptación positiva respecto al uso de una metodología asistida por computadora, en aproximadamente un $60 \%$ de la población estudiantil. 
Otro de los aspectos fundamentales que se trataron de plasmar en los grupos piloto, fue el uso del software Mathematica como un mecanismo de resolución de problemas triviales y complejos. En este sentido, los alumnos manifestaron en un $74.55 \%$ estar a favor de considerar que el software facilitó la resolución de problemas en el desarrollo de las distintas unidades temáticas de cada curso. Pese a ello, un 56.37\% manifestaron preferir una oferta educativa de un curso impartido de forma tradicional. Mediante una observación cualitativa hecha por uno de los autores de esta propuesta, asumiendo el rol de observador participante, durante al menos una de las sesiones de trabajo semanales del II semestre 2012, se considera que esta tendencia a evitar el uso de software, obedece al hecho de que esta componente aumenta el nivel de dificultad de la materia para algunos estudiantes, pues deben resolver ocasionalmente problemas con un nivel mayor de complejidad.

Naturalmente, en una metodología asistida por computadora, el objetivo a nuestro juicio no reside en continuar resolviendo los mismos problemas que se abordan con lápiz y papel como ya hemos insistido y bien lo apunta De Faria (2007). Una experiencia educativa asistida por computadora bien diseñada, debe conducir al estudiante a experimentar, conjeturar, formular hipótesis y conclusiones. Bajo esta perspectiva, los problemas a resolver no pueden ser triviales, abriendo muchas veces inclusive la posibilidad de tener varias respuestas distintas. El alumno por su cultura organizacional en la educación formal, no está acostumbrado a enfrentarse a este tipo de problemas, por lo que puede resultar muy lógico que en principio sienta poca comodidad al enfrentarse a este tipo de situaciones cognitivas. De hecho, de los estudiantes participantes, un 78.18\% nunca habían utilizado software para apoyar procesos educativos. Esto consideramos, pudo haber tenido una influencia indirecta sobre la percepción de un $36.36 \%$ de los alumnos de la muestra, que opinaron que el software se convirtió en un obstáculo para su aprendizaje. También, otros factores incidentes en esta percepción se circunscribieron en: acceso a una computadora, bases conceptuales mostradas hacia el curso y experiencias previas en el uso de sistemas operativos y aplicaciones ofimáticas, rasgos detectados mediantes las observaciones cualitativas llevadas a cabo durante el semestre.

En cuanto a algunas recomendaciones específicas proporcionadas por la población estudiantil sobre esta experiencia se destaca:

- Una mejor inducción al uso del software Mathematica.

- En los laboratorios se brinde un mayor tiempo para la solución de los problemas.

- Se dé la opción de resolver los exámenes únicamente en papel (posiblemente este hecho al observar el nivel de dificultad de las preguntas en un examen asistido por ordenador).

- No enfatizar tanto en el lenguaje de programación de la herramienta.

- Destinar mayor tiempo de clase para resolver problemas triviales.

En general, se concluye que la experiencia tuvo aproximadamente un $60 \%$ de acogida en la población estudiantil, desde nuestro punto de vista este porcentaje es muy positivo pues se desarrollaron en los cursos problemas con un nivel de dificultad y de profundización mayor, en comparación con semestres anteriores y los otros grupos control (grupos abiertos durante el II semestre 2013 impartidos por otros docentes). Esto desde luego, generó oposición en algunos estudiantes al compararse con alumnos de otros docentes y en algunos casos al comparar el curso con el recibido en otros semestres. Nos parece que las reacciones son naturales al tratar con estudiantes inexpertos en cuanto al uso de este tipo de metodologías. 
Desde un punto de vista personal, creemos que esta experiencia debería replicarse nuevamente, pues presenta las siguientes ventajas:

- Un aprendizaje de mayor nivel de profundidad en muchas de las unidades temáticas, pues al incorporar el uso de software se brinda la posibilidad de resolver problemas más significativos.

- Se logra un cambio metodológico que bien canalizado, podría tener un efecto positivo en el nivel de motivación de la población estudiantil.

- Se logran mejores aproximaciones de la vinculación de los cursos con el área disciplinar del estudiante.

- El alumno aprende a utilizar una herramienta que le servirá en el transcurso de su carrera y en su vida profesional, como un mecanismo de investigación científica.

Así mismo, con la intención de mejorar la experiencia en futuras versiones se sugieren las siguientes recomendaciones:

- Se alterne semanalmente una clase en el laboratorio y otra en el aula.

- Se limiten los cupos de matrícula a 15 estudiantes, pues con 20 o más alumnos se dificulta mucho poder otorgar un tratamiento más personalizado.

- Apoyar un proceso de capacitación docente sobre el uso del software Mathematica en el campo de las estructuras discretas y el álgebra lineal.

\subsection{Conclusiones}

La enseñanza y el aprendizaje de la matemática asistida por computadora requiere una planificación seria en las dimensiones: metodológica, recursos y evaluación. Una experiencia docente bien mediada orienta los esfuerzos a potencializar el aprendizaje a través del uso de la tecnología, que se hace invisible hacia la consecución de objetivos pedagógicos.

La tarea demanda esfuerzos de cambio administrativos y académicos empujados por iniciativas de innovación como la que hemos propuesto mediante el presente trabajo de investigación. La experiencia nos ha mostrado en este campo, un avance lento pero prometedor hacia un perfeccionamiento metodológico y de recursos didácticos.

No creemos que el computador sea la respuesta a una mejora sustantiva de los procesos educativos, creemos que como un medio más, puede favorecer la estructuración de ambientes de aprendizaje de carácter exploratorio como un complemento dentro de la diversidad que enfrentan las instituciones de educación superior. 


\section{Bibliografía}

[1] Alpízar, M. (2007). Herramientas tecnológicas en el proceso de enseñanza y aprendizaje de la estadística. Cuadernos de investigación y formación en educación matemática, 2 (3), 99-118.

[2] Astorga, A. y Sánchez, A. (1999). Enseñanza de la Matemática Asistida por Computadora: Experiencia del Instituto Tecnológico de Costa Rica. Memorias del Primer Congreso Internacional de Enseñanza de la Matemática Asistida por Computadora, 1(1), 197-206.

[3] Coicaud, S. (2010). Educación a distancia: tecnologías y acceso a la educación superior. Argentina: Editorial Biblos.

[4] Córica, J y Dinerstain, P. (2009). Diseño curricular y nuevas generaciones, incorporando a la generación .net. Argentina: Editorial Virtual Argentina.

[5] De Faria, E. (2006). Matemáticas y nuevas tecnologías en Costa Rica. En Ricardo Cantoral Uriza, Olda Covián Chávez et al. (Comps.), Investigaciones sobre enseñanza y aprendizaje de las matemáticas (pp. 709-732). México: Ediciones Díaz de Santos, S.A.

[6] De Faria, E. (2007). Tecnología digital y resolución de problemas. Cuadernos de investigación y formación en educación matemática, 2 (3), 119-131.

[7] Gamboa, R. (2007). Uso de la tecnología en la enseñanza de las matemáticas. Cuadernos de investigación y formación en educación matemática, 2 (3), 11-44.

[8] Gardner, H. (2001). La inteligencia reformulada. España: Espasa Libros, S.L.U.

[9] Piscitelli, A. (2010). Edupunk, maestros ignorantes, educación invisible y el Proyecto Facebook. En Alejandro Piscitelli, Iván Adaime, Inés Binder (Comps.), El proyecto Facebook y la posuniversidad (pp. 3-19). España: Editorial Ariel.

[10] Vílchez, E. (2012). Álgebra lineal apoyada con Mathematica. Costa Rica: Editorial Tecnológica.

[11] Vílchez, E. (2013). Estructuras Discretas con Mathematica. Costa Rica: Escuela de Informática de la Universidad Nacional de Costa Rica.

[12] Wolfram Research (2013). Historia y orígenes. http://www.wolfram.com/company/mathematicahistory.es.html. Consultado 13/02/2013. 


\section{Apéndice A}

\section{Cuestionario aplicado a la población estudiantil}

El presente cuestionario tiene como objetivo recolectar información que contribuya con el establecimiento de una percepción estudiantil, sobre la eficiencia del uso de software para apoyar procesos de aprendizaje e investigación, con la intención de determinar la factibilidad de su continuidad de uso, en futuros semestres.

\section{A. Información general}

1. Género: ( ) Masculino ( ) Femenino

2. Edad:

3. Carrera que cursa:

4. Universidad donde lleva esta carrera:

5. Nombre del curso matriculado:

\section{B. Valoración del uso de software.}

Marque con una $\mathbf{x}$ dentro de la casilla correspondiente a la opción que más se adecua a cada una de las afirmaciones presentadas. Lea cuidadosamente antes de contestar. 


\begin{tabular}{|c|c|c|c|c|c|}
\hline $\begin{array}{l}\text { Eficiencia del uso de } \\
\text { software }\end{array}$ & $\begin{array}{c}1 . \\
\text { Muy de } \\
\text { acuerdo }\end{array}$ & $\begin{array}{c}2 . \\
\text { De acuerdo }\end{array}$ & \begin{tabular}{c|}
3. \\
Medianamente \\
de acuerdo
\end{tabular} & $\begin{array}{c}4 . \\
\text { En } \\
\text { desacuerdo }\end{array}$ & $\begin{array}{c}5 . \\
\text { Muy en } \\
\text { desacuerdo }\end{array}$ \\
\hline $\begin{array}{l}\text { 6. Los procesos de } \\
\text { enseñanza mejoraron } \\
\text { con el uso de soft- } \\
\text { ware. }\end{array}$ & & & & & \\
\hline $\begin{array}{l}\text { 7. Los procesos de } \\
\text { aprendizaje no mejo- } \\
\text { raron con el uso de } \\
\text { software. }\end{array}$ & & & & & \\
\hline $\begin{array}{l}\text { 8. El software facil- } \\
\text { itó la resolución de } \\
\text { problemas. }\end{array}$ & & & & & \\
\hline $\begin{array}{l}\text { 9. Prefiero que se } \\
\text { use una metodología } \\
\text { más tradicional al } \\
\text { impartir el curso. }\end{array}$ & & & & & \\
\hline $\begin{array}{l}\text { 10. No considero que } \\
\text { el software Mathe- } \\
\text { matica ayude en los } \\
\text { procesos de investi- } \\
\text { gación (exploración y } \\
\text { construcción de con- } \\
\text { jeturas). }\end{array}$ & & & & & \\
\hline $\begin{array}{l}\text { 11. El software se } \\
\text { convirtió en un ob- } \\
\text { stáculo en mi apren- } \\
\text { dizaje. }\end{array}$ & & & & & \\
\hline $\begin{array}{l}\text { 12. El curso no } \\
\text { mejoró en cuanto a } \\
\text { calidad académica } \\
\text { al utilizar una } \\
\text { metodología asistida } \\
\text { por computadora. }\end{array}$ & & & & & \\
\hline $\begin{array}{l}\text { 13. Nunca habia } \\
\text { utilizado software } \\
\text { para complemen- } \\
\text { tar los procesos } \\
\text { de enseñanza y } \\
\text { aprendizaje. }\end{array}$ & & & & & \\
\hline
\end{tabular}

14. ¿Estaría dispuesto a recomendar a otros estudiantes llevar el curso con una metodología asistida por computadora?, explique las razones.

15. ¿Qué recomendaciones brindaría para mejorar la calidad académica y pedagógica del curso? 
Muchas gracias por su colaboración. 